\title{
INFORME
}

\section{Medidas frente al cambio climático y para la transición hacia un nuevo modelo energético en Andalucía}

\section{INTRODUCGIÓN}

El objeto del presente comentario es la Ley 8/2018, de 8 de octubre, de medidas frente al cambio climático y para la transición hacia un nuevo modelo energético en Andalucía. Publicada en el BOJA 199, de 15 de octubre, entrará en vigor a los tres meses de la publicación, salvo las previsiones relativas al Sistema Andaluz de Emisiones Registradas, que producirán efectos a la entrada en vigor del Reglamento que regule el Sistema; y las relativas al artículo 42.2 (obligaciones en la modalidad de reducción de emisiones del Sistema), que producirán efectos al año de la entrada en vigor de dicho Reglamento (disposición final séptima).

Componen la Ley la exposición de motivos, 64 artículos, cuatro disposiciones adicionales, siete disposiciones finales ${ }^{2} \mathrm{y}$ un Anexo en el que se establece el sentido de determinados términos que emplea la Ley ${ }^{3}$. A continuación, se reseñarán sus aspectos más destacados, no sin antes subrayar que se trata, sin duda, de una ley trascendental, aprobada por unanimidad, que, centrada en los sectores difusos, se anticipa a la legislación estatal y sitúa a Andalucía, junto con la Comunidad Autónoma de Cataluña ${ }^{4}$, en la vanguardia de la lucha frente al cambio climático y de la transición energética.

Esta sección ha sido elaborada por MARÍA DEL CARMEN NÚÑEZ LOZANO, Catedrática de Derecho Administrativo, Centro de Investigación en Patrimonio Histórico, Cultural y Natural, Universidad de Huelva. Proyecto "Nuevo Derecho Urbanístico: simplificación, sostenibilidad, rehabilitación" (DER2017-84387-P).

2 La primera modifica la Ley $7 / 2007$, de 9 de julio, de Gestión Integrada de la Calidad Ambiental. La segunda, la Ley 1/1994, de 11 de enero, de Ordenación del Territorio de la Comunidad Autónoma de Andalucía. La tercera, la Ley 2/1989, de 18 de julio, por la que se aprueba el Inventario de Espacios Naturales Protegidos de Andalucía y se establecen medidas adicionales para su protección. La cuarta, la Ley de 9/2010, de 30 de julio, de Aguas de Andalucía. La quinta se refiere al cambio climático como objetivo específico de los Planes Estadísticos y Cartográficos de Andalucía. Las disposiciones finales tercera y cuarta no figuraban en el texto del Proyecto de Ley y fueron introducidas durante la tramitación parlamentaria. lada".

3 Y, curiosamente, de otros que no utiliza la Ley: "combustibles alternativos" y "población vincu-

4 Pionera con la aprobación de la Ley 16/2017, de 1 de agosto, del cambio climático. 


\section{FINALIDAD DE LA LEY, ÁMBITO DE APLICACIÓN Y PRINCI- PIOS RECTORES}

La finalidad de la Ley es, según el artículo 1, "la lucha frente al cambio climático y hacia un nuevo modelo energético en Andalucía". Su objeto se precisa mediante la enumeración de una serie de acciones, propósitos y metas de carácter diverso, que responden a los perfiles de la mitigación ${ }^{5}$; de la adaptación ${ }^{6}$; del conocimiento ${ }^{7}$; o que tienen un carácter más reflexivo en orden a precisar el marco normativo y asegurar la participación ciudadana y la información pública ${ }^{8}$ (artículo 1).

La Ley se aplica a "aquellos sectores y actividades de cualquier naturaleza que incidan en la consecución de los fines marcados" en la Ley. Se excluyen "las emisiones de los gases incluidos en el ámbito de la Ley 1/2005, de 9 de marzo, por la que se regula el régimen del comercio de derechos de emisión de gases de efecto invernadero", lo que a mi juicio no es por completo correcto, debiéndose entender que se excluyen estas emisiones en tanto sean producidas por las actividades incluidas en el Anexo I de dicha Ley.

Por último, los principios rectores son los recogidos en el artículo 4: "a) Precaución ante los riesgos potenciales no conocidos. b) Prevención de los riesgos conocidos. c) Mejora continua, de acuerdo con el mejor conocimiento científico disponible. d) Desarrollo sostenible, basado en la protección del medioambiente, el desarrollo so-

5 “a) Establecer los objetivos y medidas de reducción de las emisiones de gases de efecto invernadero e incrementar la capacidad de los sumideros de $\mathrm{CO} 2$, todo ello teniendo en cuenta los objetivos que al respecto marquen la Unión Europea y el Gobierno de España, para llevar a cabo una transformación ordenada de nuestra economía hacia una economía baja en carbono y resiliente al clima [...] d) Impulsar la transición energética justa hacia un futuro modelo social, económico y ambiental en el que el consumo de combustibles fósiles tienda a ser nulo, basada en la promoción de un sistema energético andaluz descentralizado, democrático y sostenible cuya energía provenga de fuentes de energía renovables y preferentemente de proximidad [...] i) Fijar los objetivos de reducción de emisiones difusas en Andalucía.

6 "b) Reducir el riesgo de los impactos del cambio climático, minimizando sus efectos [...] e) Reducir la vulnerabilidad de la sociedad andaluza ante los impactos adversos del cambio climático, así como crear los instrumentos necesarios que ayuden a reforzar las capacidades públicas de respuesta a estos impactos [...] f) La adaptación de los sectores productivos e incorporar el análisis de la resiliencia a los efectos adversos del cambio climático en la planificación del territorio, los sectores y actividades, las infraestructuras y las edificaciones". Ha de observarse que "la adaptación de los sectores productivos" puede interpretarse en clave de mitigación o en clave de adaptación.

7 “g) Fomentar la educación, investigación, el desarrollo y la innovación en materia de adaptación y mitigación del cambio climático [...] j) El fomento y la difusión del mejor conocimiento técnico-científico en materia climática y la incorporación de las externalidades en los procesos de análisis coste-beneficio".

8 “c) Definir, en el ámbito de las competencias de la Administración de la Junta de Andalucía, el marco normativo para la incorporación de la lucha contra el cambio climático en las principales políticas públicas afectadas, de acuerdo con los conocimientos técnicos y científicos disponibles [...] h) Promover la participación ciudadana y la información pública de la sociedad andaluza en la elaboración y evaluación de las políticas contenidas en la presente ley". 
cial y el económico. e) Protección de la competitividad de la economía andaluza. f) Coordinación y cooperación administrativa. g) Responsabilidad compartida de las Administraciones públicas, de las empresas y de la sociedad en general. h) Participación pública e información ciudadana". Como se aprecia, junto con genuinos principios del Derecho Ambiental -sin duda los más importantes- se incluyen otras menciones que no lo son propiamente ${ }^{9}$ o que son elementos de principios ya enunciados ${ }^{10}$.

\section{COMPETENCIAS Y ORGANIZAGIÓN ADMINISTRATIVAS}

A estas materias se dedica el Título primero de la Ley que, además de enunciar los deberes de cooperación y colaboración de las administraciones competentes (artículo 5), anuncia la creación por el Consejo de Gobierno de la Comisión Interdepartamental de Cambio Climático (artículo 6) y crea la Oficina Andaluza de Cambio Climático (artículo 7).

En la Comisión deben estar representadas "todas las Consejerías competentes en las materias correspondientes a las áreas estratégicas para la mitigación de emisiones y para la adaptación, a través de personas titulares de órganos directivos con rango al menos de viceconsejería, o en su caso, de secretaría general" (artículo 6.1). Y la Comisión, a su vez, contará con representación en el Consejo Andaluz del Clima (artículo 6.4). Sus funciones, en relación con el Plan Andaluz de Acción por el Clima, serán: “a) Impulsar las políticas de mitigación de emisiones, adaptación y comunicación del cambio climático en la Junta de Andalucía. b) Coordinar la actuación de las Consejerías de la Junta de Andalucía en la lucha contra el cambio climático. c) Supervisar que se mantenga el equilibrio económico, ambiental y social entre las acciones de mitigación, adaptación y comunicación. d) Emitir informe de valoración con carácter previo a la aprobación del Plan Andaluz de Acción por el Clima y sus revisiones para el fomento de un nuevo modelo energético. e) Colaborar, a instancias de la Consejería competente, en la redacción del Plan Andaluz de Acción por el Clima. f) Impulsar la transferencia de información, estableciendo canales de comunicación desde los centros productores de conocimiento hasta los responsables de gestión" (artículo 6.3).

Por su parte, la Oficina, con naturaleza de unidad administrativa, se adscribe a la Consejería competente en materia de cambio climático, señalándose que "a) Elaborará los informes de seguimiento de los objetivos, medidas y actuaciones establecidas en el Plan Andaluz de Acción por el Clima, proponiendo, en su caso, los me-

\footnotetext{
9 "Coordinación y cooperación administrativa" y "participación pública e información ciudadana".

10 "La mejora continua, de acuerdo con el mejor conocimiento científico disponible", puede insertarse en el desarrollo sostenible, al igual que la "protección de la competitividad de la economía andaluza".
} 
canismos de corrección que sean necesarios. b) Elaborará la propuesta de Inventario Andaluz de Emisiones de Gases de Efecto Invernadero del artículo 18. c) Realizará actividades de formación y asesoramiento para el desarrollo y planificación de actividades en materia de mitigación, comunicación y adaptación al cambio climático. d) Formulará los criterios para la elaboración de proyectos de fijación de carbono en espacios naturales protegidos. e) Servirá de plataforma para la transferencia de información, estableciendo canales de comunicación desde los centros productores de conocimiento hasta los responsables de gestión" (artículo 7.2).

\section{PLANIFICACIÓN FRENTE AL GAMBIO CLIMÁTICO E IMPUL- SO PARA LA TRANSICIÓN ENERGÉTICA}

La Ley contempla planes municipales contra el cambio climático (artículo 15) y regula el Plan Andaluz de Acción por el Clima (artículos 8 y siguientes). También prevé los denominados Escenarios Climáticos de Andalucía (artículo 17) y el Inventario Andaluz de Emisiones de Gases de Efecto Invernadero (artículo 18), que se caracterizan, todos ellos, como "instrumentos de referencia para la planificación"

El plan de cabecera es el Plan Andaluz de Acción por el Clima, que tiene la consideración de plan con incidencia en la ordenación del territorio (artículo 8.2) y cuyas determinaciones son obligatorias para todas las administraciones y para todas las personas jurídicas o físicas titulares de actividades incluidas en el ámbito de la Ley (artículo 8.1). En su formulación se ha de procurar "la integración efectiva en la planificación autonómica y local de las acciones de mitigación, adaptación y comunicación del cambio climático, y que se aprovechen las sinergias entre dichas acciones" (artículo 9.1). Su contenido mínimo es el que recoge el artículo 9.2, destacando las menciones sobre información (letra d), participación (letra e), estrategias en materia de mitigación y de adaptación (letra f), acciones de comunicación, participación, formación y educación ambiental (letra h), medidas para colectivos especialmente vulnerables a los efectos del cambio climático (letra k), marco estratégico de transición hacia un nuevo modelo energético (letra l) y colaboración, fomento y promoción para la adaptación del tejido empresarial y productivo andaluz (letra n). Se prevé que el Plan incluya un Programa de Mitigación de Emisiones para la Transición Energética (artículo 10), un Programa de Adaptación (artículo 11) y un Programa de Comunicación y Participación (artículo 12). Habrá de estar aprobado en el plazo de un año desde la entrada en vigor de la Ley, plazo sin duda breve si se tiene en cuenta que el plazo de que disponen las Consejerías para aportar información a la

11 El reglamento del Inventario debe ser aprobado en el plazo máximo de dos años desde la entrada en vigor de la Ley (apartado tercero de la disposición adicional tercera). 
Consejería competente en materia de cambio climático es de seis meses (disposición adicional primera).

En cuanto a los planes municipales contra el cambio climático, la Ley dispone su elaboración y aprobación en el ámbito de las competencias propias que el artículo 9 de la Ley 5/2010, de 11 de junio, de Autonomía Local de Andalucía, atribuye a los municipios y en el marco de las determinaciones del Plan Andaluz de Acción por el Clima (artículo 15.1). Recaen sobre las áreas estratégicas en materia de mitigación de emisiones y adaptación que establecen los artículos 10.2 y 11.2, respectivamente, de la Ley (artículo 15.2). Con el contenido mínimo que prescribe el artículo 15.2, pueden elaborarse y aprobarse a través de las entidades e instrumentos de cooperación territorial que se enumeran en el artículo 62 de la Ley 5/2010 (artículo 15.5). La aprobación de los planes debe tener lugar dentro del año siguiente a la aprobación del Plan Andaluz de Acción por el Clima (disposición adicional segunda).

\section{ADAPTAGIÓN AL GAMBIO GLIMÁTICO}

El Título tercero de la Ley, sobre la adaptación al cambio climático, regula la integración de la adaptación al cambio climático en los instrumentos de planificación y crea el Registro de la huella hídrica de productos, servicios y organizaciones.

La integración de la adaptación al cambio climático en los instrumentos de planificación la resuelve la Ley estableciendo que "las actividades de planificación autonómica y local relativas a las áreas estratégicas para la adaptación al cambio climático establecidas en el artículo 11 tendrán [...] la consideración de planes con incidencia en materia de cambio climático" (artículo 19.1), debiendo en consecuencia incluir los contenidos que señala el artículo $19.2^{12}$, a cuyo efecto el artículo 20 enumera los impactos que al menos han de considerarse según el área estratégica de adaptación que se trate.

El Registro de la huella hídrica de productos, servicios y organizaciones tiene por objeto "la inscripción voluntaria de la huella de hídrica de los productos, servicios y organizaciones, entendida como herramienta para calcular y comunicar el consumo de recursos hídricos asociados al ciclo de vida de un producto, servicio $u$ organización" (artículo 21.1). Pueden inscribirse "las personas físicas o jurídicas tanto públicas como privadas que produzcan, distribuyan o comercialicen un producto o servicio en la Comunidad Autónoma de Andalucía” (artículo 21.2) y la inscripción otorga "el derecho a utilizar el logotipo de la huella hídrica en el establecimiento

12 El expresado en la letra b) - "Las disposiciones necesarias para fomentar la baja emisión de gases de efecto invernadero y prevenir los efectos del cambio climático a medio y largo plazo"- guarda más relación con la mitigación que con la adaptación. 
o en la etiqueta del producto" (artículo 21.5). Los productos, en particular, "deben incorporar una evaluación de la huella hídrica visible en el etiquetado y el embalaje" (artículo 21.6).

\section{MEJORA DEL CONOGIMIENTO Y PARTICIPACIÓN PÚBLICA}

Para la mejora del conocimiento en la materia, la Ley crea la Red de Observatorios de Cambio Climático de Andalucía (artículo 22) y prevé la imposición de obligaciones relacionadas con la transmisión de información a la administración, así como la incorporación del cambio climático como objetivo específico de los planes estadísticos de Andalucía (artículo 23.2 y 3), precisando que la información en la materia será pública (artículo 23.4). También contempla medidas de fomento de la investigación, el desarrollo y la información (artículo 24) y el tratamiento de la materia en los estudios universitarios y no universitarios (artículo 25).

Por lo que respecta a la participación y a la transparencia, se crea el Consejo Andaluz del Clima "para facilitar la participación de la sociedad civil en el diseño y seguimiento de las políticas en materia de cambio climático" (artículo 26.1); se prevén "acciones y campañas que tengan por finalidad informar y concienciar a la ciudadanía en materia de cambio climático", así como canales de información "para los agentes económicos y sociales implicados, la ciudadanía y la Administración pública" (artículo 27); se dispone específicamente el impulso de "los canales de difusión de la información en esta materia [...] con la finalidad de fomentar la participación de la ciudadanía en el diseño de las políticas públicas de mitigación y adaptación" (artículo 28.1); se efectúa una remisión a la legislación de transparencia y de acceso a la información ambiental en lo concerniente al derecho de acceso público a la información (artículo 28.2); y se contempla la remisión bianual al Parlamento de una memoria con la información sobre el grado de cumplimiento de los objetivos de la Ley, que debe ser publicada en el Informe de Medio Ambiente de Andalucía y, también, ser accesible por vía telemática (artículo 29).

\section{GONTRATAGIÓN PÚBLICA Y PRESUPUESTOS}

En materia de contratación pública, la Ley ordena a las entidades del sector público a las que se aplica la Ley 9/2017, de 8 de noviembre, de Contratos del Sector Público, que promuevan una serie de medidas de "contratación pública verde", que es el nombre del artículo 30. El artículo 53, por otra parte, contempla la posibilidad de exigir la huella de carbono en las licitaciones que lleven a cabo la Administración de la Junta de Andalucía y sus entes instrumentales, posibilidad que devendrá en obligación una vez que transcurran dos años desde la entrada en vigor de la Ley. 
En materia de presupuestos, el artículo 31 prevé la incorporación al Proyecto de Ley de Presupuestos de la Comunidad Autónoma de un "informe sobre la incidencia de los indicadores presupuestarios en el cambio climático”. El artículo 32, por su parte, contempla "un informe anual sobre el presupuesto en materia de cambio climático y su grado de ejecución”, informe que ha de hacerse público.

\section{MITIGACIÓN DE EMISIONES}

En el extenso Título VII se aborda la mitigación de emisiones. Consta de cuatro capítulos en los que se regulan los objetivos y medidas de mitigación de emisiones, el Sistema Andaluz de Emisiones Registradas, el Sistema Andaluz de Compensación de Emisiones y la huella de carbono de productos y servicios.

Los objetivos de mitigación de emisiones difusas se fijan, "como mínimo, en función de los objetivos de la Unión Europea y de la normativa básica estatal, adoptando el criterio de reparto proporcional del esfuerzo según el tramo que le corresponda al PIB relativo a la Comunidad Autónoma, y aplicando el esfuerzo así determinado a las emisiones de CO2 equivalentes per cápita, o, en su caso, el criterio que en el futuro le pueda sustituir" (artículo 33.1). En concreto, se establece como objetivo para 2030 la reducción como mínimo del 18\% de emisiones difusas de gases de efecto invernadero por habitante con respecto a 2005, adoptándose el valor de 33.321 kilotoneladas equivalentes de CO2 para este año 2005, lo que se corresponde con 4,21 toneladas equivalentes de CO2 per cápita (artículo 33.2). Los objetivos para periodos posteriores los fijará el Gobierno con arreglo a los parámetros que cita el artículo 33.3, pudiendo revisar el objetivo si cambiaran las circunstancias a que se refieren estos parámetros (artículo 33.3).

Los objetivos para la transición hacia un nuevo modelo energético ${ }^{13}$ los fija el artículo 34, que apela también a la Ley 2/2007, de 27 de marzo, de fomento de las energías renovables y del ahorro y la eficiencia energética de Andalucía. Se

13 Se trata de "un modelo energético renovable y descarbonizado, neutro en emisiones de gases de efecto invernadero, que reduzca la vulnerabilidad del sistema energético andaluz y garantice el derecho de acceso a la energía como bien común". Las medidas encaminadas a la transición energética hacia este modelo deben enfocarse a: "a) Impulsar políticas de ahorro y eficiencia energéticos, con el objetivo de reducir el consumo tendencial de energía primaria en el año 2030, como mínimo el 30\%, excluyendo los usos no energéticos. b) Promover las energías renovables y un modelo energético en el que el consumo de combustibles fósiles tienda a ser nulo, para que en 2030 se pueda aportar con energías renovables, como mínimo, el 35\% del consumo final bruto de energía. c) Adoptar medidas de carácter normativo que favorezcan el autoconsumo energético a partir de energías renovables y la participación de actores locales en la producción y distribución de energía renovable, así como la simplificación normativa en la tramitación de los proyectos renovables. d) Difundir los principios del nuevo modelo energético a través de actuaciones de sensibilización e informar a la ciudadanía, incluyendo acciones formativas dirigidas al sistema educativo andaluz" (artículo 34.1). 
pretende reducir el consumo tendencial de energía primaria en el año 2030, como mínimo el 30\%, excluyendo los usos no energéticos; y que en 2030 se pueda aportar con energías renovables, como mínimo, el 35\% del consumo final bruto de energía. Igualmente, los objetivos para periodos posteriores los fijará el Gobierno con arreglo a los parámetros que cita el artículo 34.2, pudiendo revisar el objetivo si cambiaran las circunstancias a que se refieren estos parámetros (artículo 34.2).

Para la mitigación de emisiones se enuncian medidas de aplicación transversal en todas las áreas estratégicas de mitigación a que se refiere el artículo 10.2 (artículo 35). Y también medidas específicas por áreas estratégicas, excepto el área de administraciones públicas (artículo 36). Todas ellas deben incluirse en el Programa de Mitigación de Emisiones para la Transición Energética.

La Ley regula distintos instrumentos para la reducción de emisiones de gases de efecto invernadero, uno obligatorio (el Sistema Andaluz de Emisiones Registradas) y otros voluntarios (el Sistema Andaluz de Compensación de Emisiones, la calificación de Municipio de Baja Emisión de Carbono y el Registro de la huella de carbono de productos y servicios). Contempla además los sumideros de carbono en espacios naturales protegidos.

El Sistema Andaluz de Emisiones Registradas obliga a los titulares de actividades públicas y privadas radicadas en Andalucía ${ }^{14}$ con un consumo eléctrico anual superior a $3 \mathrm{GWh}$ (modalidad de reducción de emisiones) o superior a $1 \mathrm{GWh}$ que no alcancen los 3 GWh (modalidad de seguimiento y notificación). Los primeros tienen las obligaciones que detalla el artículo 42.2, destacadamente las de elaborar un plan de reducción de emisiones y reducir éstas hasta el valor de referencia que se fije; los segundos tienen las obligaciones que enumera el artículo 43.2. Se consideran las emisiones directas, las indirectas y las externalizadas (artículo 44). Para la comprobación del cumplimiento de las obligaciones se crea el Registro del Sistema Andaluz de Emisiones Registradas (artículo 46). Todos los establecimientos incluidos en el Sistema deben exhibir públicamente el certificado del Sistema Andaluz de Emisiones Registradas, que acredita el cumplimiento de las obligaciones establecidas en la Ley (artículo 49) $)^{15}$.

14 Obviamente, no se aplica a las actividades a que se refiere el anexo I de la Ley 1/2005, de 9 de marzo. Pero tampoco, lógicamente, "a las actividades de defensa o seguridad nacionales, cuando ello resulte justificado por razones de seguridad pública o de protección de los intereses esenciales de seguridad del Estado" (artículo 41.5).

15 El reglamento del Sistema Andaluz de Emisiones Registradas debe ser aprobado en el plazo máximo de dos años desde la entrada en vigor de la Ley (apartado segundo de la disposición adicional tercera). 
El Sistema Andaluz de Compensación de Emisiones es, como se ha anticipado, un instrumento de carácter voluntario. Se basa en la suscripción de convenios entre las personas físicas o jurídicas y la Consejería competente en materia de cambio climático, en virtud los cuales se adquieren compromisos de seguimiento, notificación y reducción de emisiones. Los objetivos de reducción de emisiones se pueden alcanzar, parcialmente o en su totalidad, mediante la compensación de emisiones, materializada mediante la entrega de unidades de absorción generadas por la ejecución de proyectos de compensación o de autocompensación, a los que seguidamente me referiré. En todo caso, se prescribe la promoción de la adhesión a iniciativas autonómicas, estatales o de la Unión Europea que tengan por objeto la reducción o la compensación voluntaria de las emisiones de gases de efecto invernadero; y la promoción también de medidas de fomento para facilitar el cumplimiento de los compromisos que se suscriban en el marco de estas iniciativas (artículo 50). Para la comprobación del cumplimiento de lo establecido en los convenios, se crea el Registro del Sistema Andaluz de Compensación de Emisiones (artículo 51).

El artículo 37 regula los proyectos de compensación de emisiones, que tienen por objeto el incremento de la capacidad de sumidero de carbono en terrenos de dominio público. A través de estos proyectos, que deben inscribirse en el Registro del Sistema Andaluz de Compensación de Emisiones, se materializan los compromisos de compensación del Sistema Andaluz de Compensación de Emisiones. La Consejería competente en la materia debe aprobar el Catálogo de Proyectos de Compensación ${ }^{16}$ en el plazo de un año desde la entrada en vigor de la Ley. Se remite al reglamento la regulación del sistema de certificación de unidades de absorción generadas a través de los proyectos y los estándares aplicables, así como el control y seguimiento de los proyectos.

Los proyectos de autocompensación de emisiones son los que pueden ejecutar, para sus propias emisiones y en terrenos sobre los que tengan derechos reales que les autoricen para ello, las personas físicas o jurídicas titulares de actividades incluidas en el Sistema Andaluz de Compensación de Emisiones. Estos proyectos pueden pertenecer a cualquiera de las categorías enumeradas en el 37.2 y "estarán sometidos a los mismos requisitos de inscripción en el Sistema Andaluz de Emisiones Registradas y certificación de unidades de absorción que los proyectos de compensación" (artículo 38$)$.

16 El artículo 37.2 señala que "se considerarán proyectos de fijación de carbono los de forestación, reforestación, restauración y conservación de masas forestales existentes, de ecosistemas litorales, de dehesas y de monte mediterráneo, los de conservación o restauración de humedales, praderas de fanerógamas marinas u otros espacios de naturaleza análoga, y los de conservación o aumento del contenido de materia orgánica del suelo, en el ámbito de la silvicultura o de la agricultura, que formen parte del Catálogo de Proyectos de Compensación". 
Otro instrumento voluntario es la calificación de Municipio de Baja Emisión de Carbono (artículo 40). Responde a la técnica de los incentivos y es un reconocimiento que otorga la Consejería competente en materia de cambio climático "en atención a las iniciativas públicas puestas en marcha sobre esta materia a nivel municipal", siendo necesario, entre otros extremos, que se tenga aprobado el Plan Municipal contra el Cambio Climático. En cuanto a las ventajas de obtener este reconocimiento, la Ley solo menciona la posibilidad de que las bases reguladoras de ayudas o subvenciones para iniciativas relacionadas con las áreas estratégicas de mitigación incorporen la valoración de la calificación de Municipio de Baja Emisión de Carbono. Por último. el reconocimiento puede también otorgarse a las entidades de cooperación territorial del artículo 62 de la Ley 5/2010, de 11 de junio, de Autonomía de Andalucía.

La Ley crea el Registro de la huella de carbono de productos y servicios, de carácter voluntario (artículo 52). Se caracteriza como una "herramienta para calcular y comunicar el total de las emisiones de gases de efecto invernadero asociados a un producto o servicio", rigiéndose el cálculo de la huella de carbono por los estándares aceptados internacionalmente. La inscripción otorga el derecho a utilizar el logotipo de la huella de carbono en el establecimiento o en la etiqueta del producto y, por otra parte, es medio de prueba de que se dispone de la huella en orden a las licitaciones que lleven a cabo la Administración de la Junta y sus entes instrumentales. Se remiten al reglamento aspectos sustanciales de la regulación y, con discutible sistemática, se impone a la Consejería la obligación de "elaborar, con una periodicidad no superior a dos años, la huella de carbono de Andalucía, basada en el inventario de emisiones de gases de efecto invernadero".

Por último, y como ya se anticipó, el Título VI regula los sumideros de carbono en espacios naturales protegidos (artículo 39). Se prevé, en primer término, que la planificación de estos espacios incluya entre sus objetivos prioritarios el incremento de la capacidad de fijación del carbono ${ }^{17}$. En segundo lugar, se exige, para ciertos proyectos, la justificación de un balance de carbono neutro a lo largo de toda la vida del proyecto, para lo cual es posible entregar unidades de absorción provenientes de proyectos de compensación o de autocompensación.

\section{RÉGIMEN SANGIONADOR Y MEDIDAS DE EJECUCIÓN FORZOSA}

El Título VII regula esta materia, tipificando infracciones leves, graves y muy graves. Las sanciones previstas son multas (hasta 120.000 euros en caso de infracción

17 Se consideran proyectos de fijación de carbono en espacios naturales los que pertenecen a las tipologías que recoge el artículo 37.2. 
muy grave) e imposibilidad de obtener préstamos, subvenciones o ayudas públicas en materia de medioambiente (durante dos años si es infracción grave y durante 4 años si es infracción muy grave).

En cuanto al plazo máximo para notificar la resolución del procedimiento sancionador, el artículo 60 se remite a la Ley 39/2015, siendo así que ésta no establece ninguno. El plazo será por consiguiente el de 3 meses que señala por defecto el artículo 21.3 de la Ley 39/2015, que puede resultar en exceso breve.

Cabe destacar también que los plazos de prescripción de las infracciones muy graves y de las leves son de cuatro y dos años respectivamente, muy superiores a los que por defecto señala el artículo 30.1 de la Ley 40/2015. Lo mismo sucede en lo que concierne al plazo de prescripción de las sanciones (cuatro años en el caso de sanciones por infracciones muy graves y graves y dos años en el de las sanciones por infracciones leves).

Finalmente, las multas coercitivas se prevén por importe de entre 30.000 y 600 euros (artículo 64). 